\title{
Assessment of the Number and Phenotype of Macrophages in the Human BMB Samples of CML
}

\author{
Jian-Xin Song, ${ }^{1,2}$ Zi-Jin Dian, ${ }^{1}$ Yan Wen, ${ }^{3}$ Fen Mei, ${ }^{2}$ Rui-Wei Li, ${ }^{2}$ and Ya-Lian Sa \\ ${ }^{1}$ Department of Clinical Laboratory, Yunnan Provincial First People’s Hospital, Kunming, Yunnan 650032, China \\ ${ }^{2}$ Institute of Clinical and Basic Medical Sciences, Yunnan Provincial First People's Hospital, Kunming, Yunnan 650032, China \\ ${ }^{3}$ Department of Hematology, Yunnan Provincial First People's Hospital, Kunming, Yunnan 650032, China
}

Correspondence should be addressed to Ya-Lian Sa; sayalian@126.com

Received 8 August 2016; Revised 24 October 2016; Accepted 3 November 2016

Academic Editor: Paul M. Tulkens

Copyright (C) 2016 Jian-Xin Song et al. This is an open access article distributed under the Creative Commons Attribution License, which permits unrestricted use, distribution, and reproduction in any medium, provided the original work is properly cited.

\begin{abstract}
Macrophages have emerged as a key player in tumor biology. However, their number and phenotype in human bone marrow of biopsy (BMB) samples of chronic myeloid leukemia (CML) and their association with disease progression from an initial chronic phase (CP) to accelerated phase (AP) to advanced blast phase (BP) are still unclear. BMB samples from 127 CML patients and 30 patients with iron-deficiency anemia (IDA) as control group were analyzed by immunohistochemistry. The expression levels of CD68, CD163, and CD206 in BMB samples of CML patients were significantly higher than those in the patients of control group $(P<0.01)$, and we observed that their positive expression was gradually elevated during the transformation of CML-CP to AP to $\mathrm{BP}(P<0.01)$. However, the expressions of CD68, CD163, and CD206 in released group were downregulated and contrasted to these in control group; there exists statistical significance $(P<0.01)$. The percentage ratio of CD163 and CD206 to CD68 was pronounced to be increasing from CML-CP to AP to BP $(P<0.01)$. Hence, the higher proportion of CD68 ${ }^{+}, \mathrm{CD}_{163}{ }^{+}$and $\mathrm{CD} 206^{+}$ macrophages in $\mathrm{BMB}$ samples can be considered a key factor for disease progression of CML patients. Targeting macrophages, especially the M2 phenotype may help in designing therapeutic strategies for CML.
\end{abstract}

\section{Introduction}

Chronic myeloid leukemia (CML) arising from the abnormalities of hematopoietic stem/progenitor cells (HSCs/ HSPCs) remains mostly an incurable disease [1]. The stereotypical progression of CML is from a relatively benign chronic phase (CP) to accelerated phase (AP) to a fatal blast phase $(\mathrm{BP})$ resembling acute leukemia, the prognosis for which is poor, with a median survival time of only $3 \sim 4$ months [2]. Given that all treatments work much better in $\mathrm{CP}$ than advanced-phase disease, it is therefore important to explore the mechanism underlying stage progression of CML [3]. Macrophages as critical immune cells and an important member of the bone marrow microenvironments are playing key role in the innate and adaptive immune response involved in tumor biology $[4,5]$. Macrophages are very versatile cells with a high degree of plasticity taken on differential phenotype and functions under the physiological and pathological condition provided by local microenvironment. According to two extremes of a spectrum of possible macrophages polarization, macrophages are termed classically activated M1 (proinflammatory type 1) and alternatively activated M2 (anti-inflammatory type 2) subtypes [6, 7]. In the tumor area macrophages have been named tumorassociated macrophages (TAMs) $[8,9]$. It was reported that M2-like macrophages are prominently found and involved in cancer initiation, progression, and metastasis, facilitating angiogenesis, matrix breakdown, and tumor cell migration, as well as decreased tumor-infiltrating cytotoxic $\mathrm{T}$ lymphocytes (CTLs) [10-13]. However, the TAM counts and its phenotype in the BMB sample of CML patients with different phases are still unsure.

With regard to different responses to various microenvironmental stimuli during CML progression, the count and phenotype of macrophages were considered to be facilitating stage determination and the therapy target. Therefore, we 
TABLE 1: Characteristics of the CML patients in this study.

\begin{tabular}{lccc}
\hline & CML-CP & CML-AP & CML-BP \\
\hline $\begin{array}{l}\text { Number of cases } \\
\text { Gender }\end{array}$ & 30 & 21 & 15 \\
$\quad$ & 18 & 13 & 8 \\
$\quad$ Male & 12 & 8 & 7 \\
$\quad$ Female & & & \\
Age, years & & & \\
$\quad$ Median (range) & $47.60(18-76)$ & $48.05(20-79)$ & 47.67 (27-78) \\
Outcomes & & & \\
$\quad$ Released CML-CP & 30 & & \\
$\quad$ Released CML-AP & & 3 & \\
$\quad$ Relapsed CML-AP & & & 4 \\
$\quad$ Released CML-BP & & & \\
$\quad$ Relapsed CML-BP & & &
\end{tabular}

attempted to explore the expression levels of macrophages markers CD68, CD163, and CD206 detected by immunohistochemistry [14-16]. We observed a pronounced increase of $\mathrm{CD}_{6}{ }^{+}, \mathrm{CD}_{163^{+}}$, and $\mathrm{CD} 206^{+}$macrophages in the $\mathrm{BMB}$ samples of different phases of CML patients. And the percentage ratio of $\mathrm{CD} 163^{+}$and $\mathrm{CD} 206^{+}$macrophages to $\mathrm{CD} 68^{+}$ macrophages was upregulated during CML development. Thus, we speculate this may be an important step for the further transformation into AP to BP stages of CML. More importantly, our present data has proposed a novel immunological mechanism for stage progression in CML pathogenesis.

\section{Materials and Methods}

2.1. Study Approval. This study was approved by the Medical Ethics Committee of Yunnan Provincial First People's Hospital. Written informed consent was obtained from patients to authorize their participation in the study. Bone marrow biopsies were obtained from recruited adult patients seen at the Department of Hematology.

2.2. Patients. We analyzed bone marrow biopsies from 127 patients with chronic myeloid leukemia (CML) in Yunnan Provincial First People's Hospital. These 66 patients with CML received tyrosine kinase inhibitors (TKI) alone or in combination with cytosine arabinoside (Ara-C) or standard anticancer regimens; and 61 patients were followed up for 3 through 15 months. Table 1 summarizes data related to these patients. The diagnoses of CML were established on the basis of the morphological examination and cytogenesis analysis. The control group was consisted of 30 patients (12 females; 18 males) with iron-deficiency anemia (IDA). The median age was 54.5 years (range, $18-76$ years).

2.3. Bone Marrow Biopsies (BMB) Samples. Representative bone marrow trephine biopsies were performed from the posterior iliac crest.
2.4. Immunohistochemical Analysis. BMB samples were fixed by immersion in $4 \%$ buffered formalin and processed overnight at RT. Samples were sequentially decalcified with $10 \%$ buffered ethylene-diamine tetra-acetic acid (EDTA), $\mathrm{pH}$ 7.2, embedded in paraffin, and then sectioned into $4 \mu \mathrm{m}$ with a microtome [17]. The slides were baked, deparaffinized in xylene, and rehydrated through a graded alcohols series to water. Antigen retrieval was done by immersing the slides in citric acid buffer solution with $\mathrm{pH} 6.0$ and placed in autoclave at $121^{\circ} \mathrm{C}$ for $10 \mathrm{~min}$. Washes were done in Tris-buffered saline and $0.05 \%$ Tween 20 (pH 7.4). After cooling down to room temperature, sections were treated with $3 \%$ hydrogen peroxide $\left(\mathrm{H}_{2} \mathrm{O}_{2}\right)$ in phosphate-buffered saline (PBS) at room temperature for $30 \mathrm{~min}$. Slides were washed, and blocking was carried out using serum-free protein block at room temperature for $30 \mathrm{~min}$. Slides were incubated with primary antibodies (Abcam company), antiCD68 (ab955; dilution 1: 600), anti-CD163 (ab87099; dilution 1:600), and anti-CD206 (ab64693; dilution 1:600) for detection of macrophages, at $4^{\circ} \mathrm{C}$ overnight. This was followed by secondary antibody (A biotinylated link antibody and a streptavidin-horseradish peroxidase kit) for $30 \mathrm{~min}$. Finally the slides were washed with PBS and developed with DAB. All sections were counterstained with hematoxylin and then were dehydrated and mounted.

Light microscopy was used to evaluate the intensity and localization of the staining. The positive cell staining with brown in cytoplasm or cell membrane was observed. In each $\mathrm{BMB}$ section, a total number of staining cells were viewed and counted by using an objective lens (magnification $\times 40$ ) and an ocular lens (10x) in at least 5 areas. The numbers of CD $68^{+}$, $\mathrm{CD} 163^{+}$, and $\mathrm{CD} 206^{+}$cells were expressed as percentage. Immunocytochemical staining results were obtained by 2 independent observers.

2.5. Statistical Analysis. Data were presented as median (mean \pm standard error) and percentage. To determine the level of significance in differences in $\mathrm{CD}^{+} 8^{+}, \mathrm{CD}_{16} 3^{+}$, and $\mathrm{CD}_{206^{+}}$numbers between the various groups of CML patients, Kruskal-Wallis and Wilcoxon rank-Wallis test were applied [18]. Statistical analysis was performed using Statistical Package for Social Sciences (SPSS) software (version 17, SPSS, Inc, Chicago, IL, USA). Statistical significance was set at $P$ value less than 0.01 .

\section{Results}

3.1. Evaluation of CD68 in the BMB Samples of CML Patients. $\mathrm{CD} 68^{+}$macrophages were roughly satellite shaped with many cytoplasmic processes and revealed a randomly dispersed distribution in the CML bone marrow. The percentage of $\mathrm{CD} 68^{+}$macrophages was gradually elevated in $\mathrm{BMB}$ samples of CML patients with CML-CP $(27.03 \pm 3.90) \%$, CML-AP (44.64 \pm 4.84$) \%$, and CML-BP groups $(66.98 \pm 6.28) \%$ (Figures $1(\mathrm{a}), 1(\mathrm{~b}), 2(\mathrm{c})$, and $1(\mathrm{~d}))$, respectively. In contrast to the control group $(12.39 \pm 2.17) \%$, the $\mathrm{CD}^{+} 8^{+}$macrophages infiltration density in each one of CML groups was increased significantly higher $(P<0.01)$. 


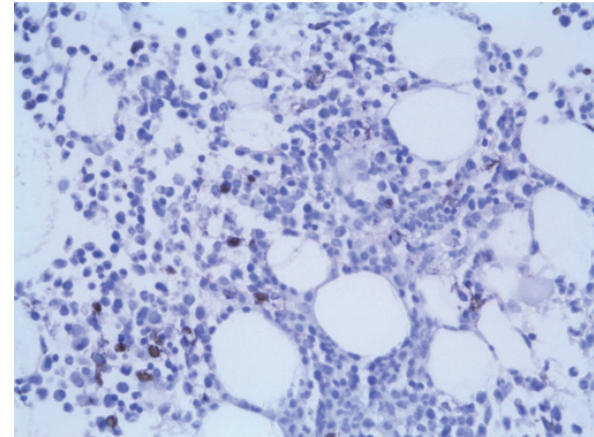

(a)

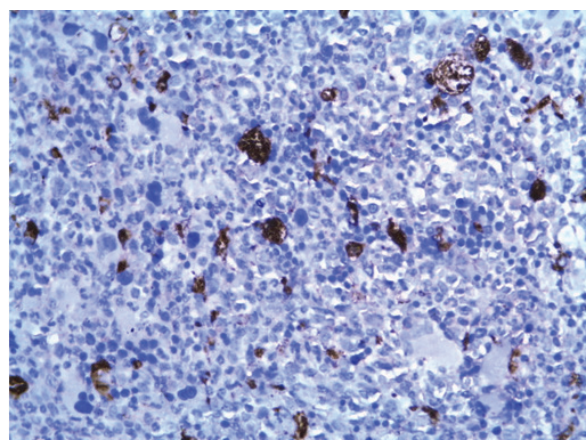

(c)

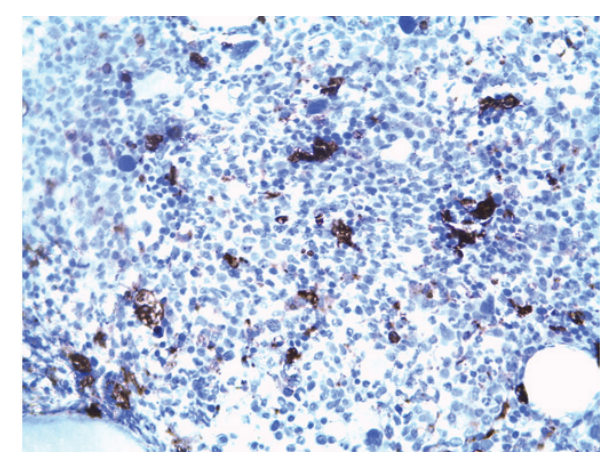

(b)

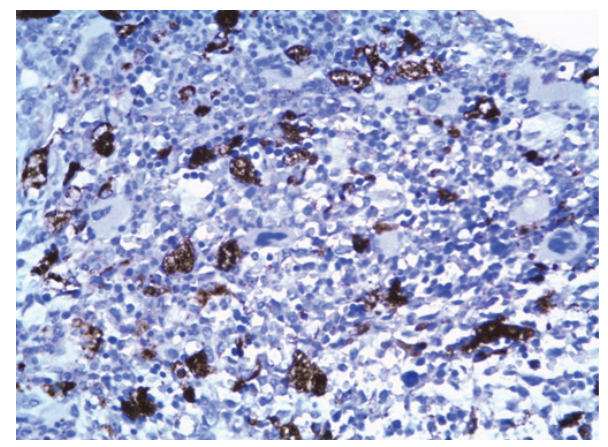

(d)

FIGURE 1: Immunohistochemical staining displaying high numbers of $\mathrm{CD} 68^{+}$macrophages in BMB samples of CML patients. CD68 was distributed in the cytoplasm of macrophages (magnification: $\times 400$ ). Interspersed CD68 expression is shown in control group (a), CML-CP (b), CML-AP (c), and CML-BP (d).

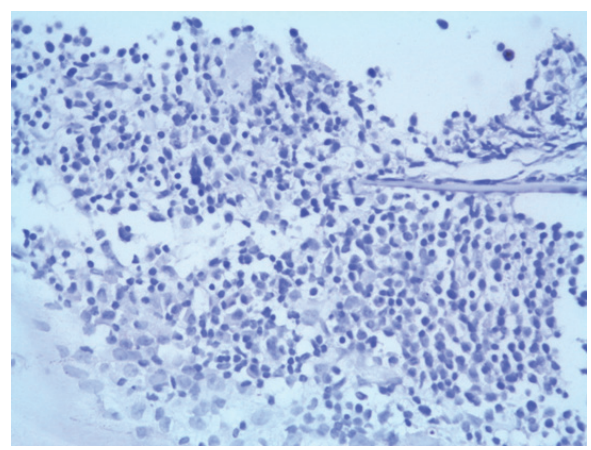

(a)

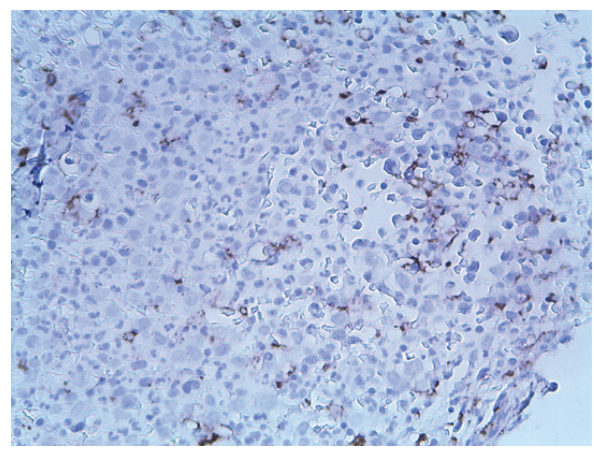

(c)

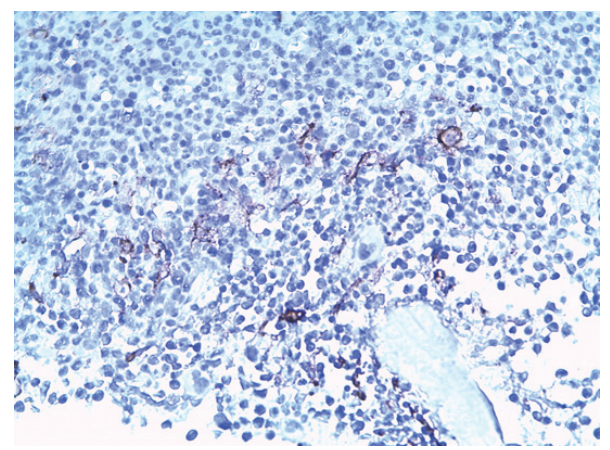

(b)

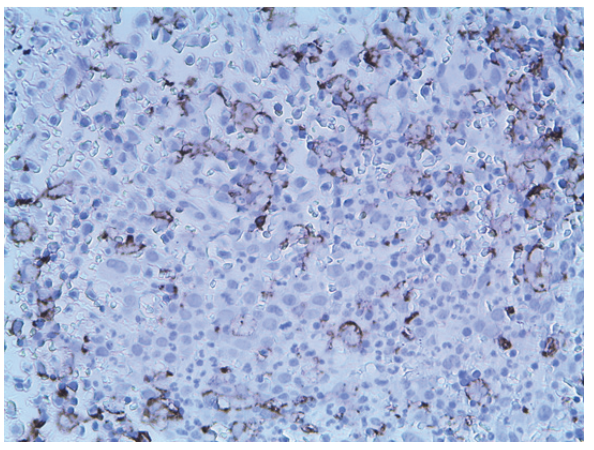

(d)

Figure 2: Immunohistochemical staining of CD163 in BMB samples of CML patients. CD163 was located in membrane of macrophages (magnification: $\times 400$ ). Scatted $\mathrm{CD}_{163^{+}}$macrophages expression is distributed in control group (a), CP-phase (b), AP-phase (c), and BP-phase (d). 


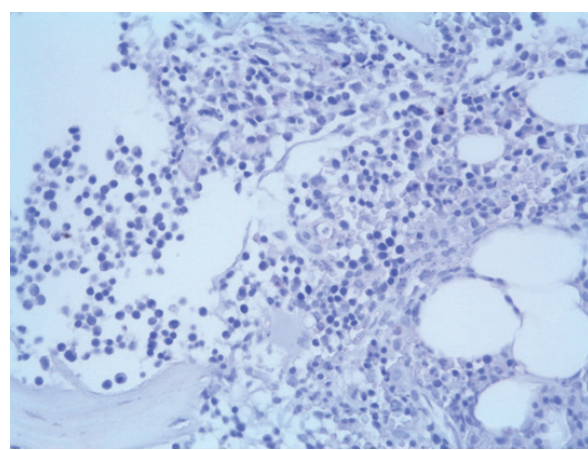

(a)

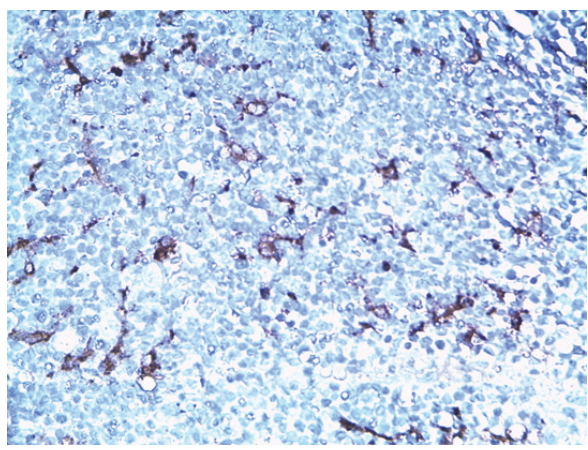

(c)

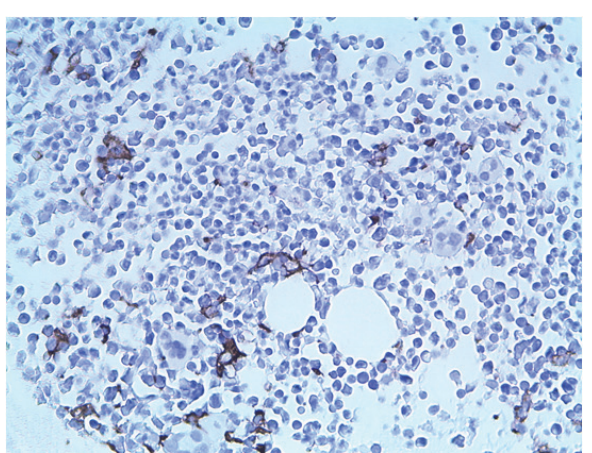

(b)

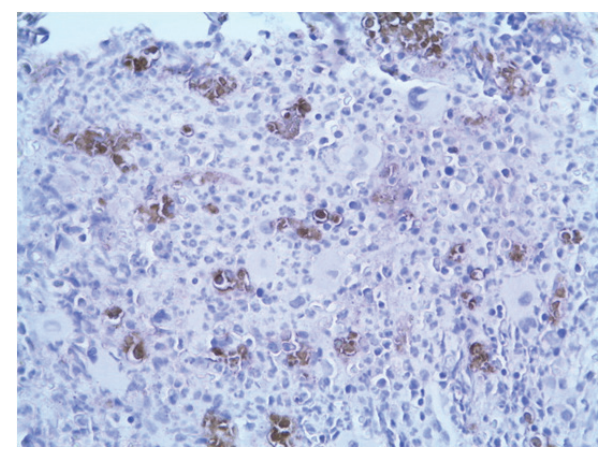

(d)

FIGURE 3: Immunohistochemical staining for CD206 in BMB samples of CML patients (magnification: $\times 400$ ). Interspersed CD206 ${ }^{+}$ macrophages expression is distributed in control group (a), CP-phase (b), AP-phase (c), and BP-phase (d).

The percentage of $\mathrm{CD} 68^{+}$macrophages was decreased in the released patients with CML-CP $(19.73 \pm 3.43) \%$, CML-AP $(25.77 \pm 4.83) \%$, and CML-BP $(43.49 \pm 4.80) \%$, respectively. In contrast to the control group, there existed statistically significant $(P<0.01)$.

3.2. CD163 Positive Expression Is Distributed in BMB Samples of CML Patients. The number of $\mathrm{CD} 163^{+}$macrophages was remarkably increased in BMB samples from CML-CP $(19.91 \pm 3.33) \%$ to CML-AP $(37.26 \pm 4.70) \%$ to CMLBP (59.97 \pm 6.79)\% (Figures 2(a), 2(b), 2(c), and 2(d)), respectively. The $\mathrm{CD}_{163^{+}}$macrophages infiltration density in BMB samples of CML patients was increased significantly higher than that of the control group $(2.71 \pm 0.82) \%(P<$ $0.01)$. The expression of $\mathrm{CD}_{163}{ }^{+}$macrophages is distributed in released patients with CML-CP $(8.73 \pm 2.00) \%$, CML$\mathrm{AP}(17.23 \pm 3.24) \%$, and CML-BP groups $(34.11 \pm 4.95) \%$, respectively. In contrast to the control group, there existed statistically significant $(P<0.01)$.

3.3. A CML Biopsy Expressed CD206 Was Upregulated. The percentage ratios of $\mathrm{CD} 206^{+}$macrophages infiltrating in the CML-CP $(20.71 \pm 3.47) \%$, CML-AP (38.57 \pm 5.12$) \%$, and CML-BP groups $(61.51 \pm 6.48) \%$ were dramatically upregulated (Figures 3(a), 3(b), 3(c), and 3(d)), respectively. Compared with the control group $(2.94 \pm 0.79) \%$, there existed statistically significant $(P<0.01)$. The percentage of CD206 ${ }^{+}$ macrophages was decreased in the released patients with
CML-CP (9.03 \pm 2.01)\%, CML-AP (17.24 \pm 3.27)\%, and CML-BP groups $(34.04 \pm 5.06) \%$, respectively. Contrasted to the control group, there existed statistically significant $(P<$ $0.01)$.

3.4. Comparisons of the Staining with $\mathrm{CD}^{+} 8^{+}, \mathrm{CD} 63^{+}$, and CD206 ${ }^{+}$Macrophages Were Shown in the BMB Samples of CML Patients. The expression levels of CD68, CD163, and CD206 in the BMB samples of CML patients were significantly increased in different phases with disease progression, especially in the CML-BP group which was infiltrated with highest frequency of macrophages (Table 2, Figure 4), when compared to that of control group.

$\mathrm{CD} 8^{+}$macrophages were usually outnumbered by $\mathrm{CD}_{163}{ }^{+}$macrophages as well as $\mathrm{CD} 206^{+}$macrophages at the same stage of CML; however, there was no difference between the positive expression of CD163 and CD206 $(P>0.01)$.

3.5. The Positive Percentage Ratios of CD163 and CD206 to CD68 Were Upregulated with the Progression of CML. The percentage ratios of CD163+ and CD206+ macrophages to CD68+ macrophages in CML-CP, CML-AP, and CML$\mathrm{BP}$ were significantly higher than those in control group, respectively (Figures 5(a) and 5(b)). These data imply that activation of macrophages in BMB samples towards the M2-like phenotype associated with the phase's development of CML. However, there was no difference between the 
TABLE 2: The positive percentages of the expression among CD68, CD163, and CD206 in BMB samples of CML patients with different phases (percentage, mean $\pm \mathrm{SD}$ ).

\begin{tabular}{|c|c|c|c|c|c|c|}
\hline Group & Cases & CD68 & CD163 & CD206 & CD163/CD68 (\%) & CD206/CD68 (\%) \\
\hline Control & 30 & $12.39 \pm 2.17$ & $2.71 \pm 0.82$ & $2.94 \pm 0.79$ & 21.90 & 23.73 \\
\hline CML-CP & 30 & $27.03 \pm 3.90$ & $19.91 \pm 3.33$ & $20.71 \pm 3.47$ & 73.68 & 76.62 \\
\hline CML-AP & 21 & $44.64 \pm 4.84$ & $37.26 \pm 4.70$ & $38.57 \pm 5.12$ & 83.63 & 86.40 \\
\hline CML-BP & 15 & $66.98 \pm 6.28$ & $59.97 \pm 6.79$ & $61.51 \pm 6.48$ & 89.23 & 91.83 \\
\hline Released CML-CP & 30 & $19.73 \pm 3.43$ & $8.73 \pm 2.00$ & $9.03 \pm 2.01$ & 44.37 & 45.77 \\
\hline Released CML-AP & 15 & $25.77 \pm 4.83^{*}$ & $17.23 \pm 3.24$ & $17.24 \pm 3.27$ & 66.84 & 66.90 \\
\hline Relapsed CML-AP & 3 & $48.80 \pm 8.15$ & $41.67 \pm 7.08$ & $41.67 \pm 7.03$ & 85.38 & 85.39 \\
\hline Released CML-BP & 9 & $43.49 \pm 4.80$ & $34.11 \pm 4.95$ & $34.04 \pm 5.06$ & 78.44 & 78.27 \\
\hline Relapsed CML-BP & 4 & $78.00 \pm 4.49$ & $66.85 \pm 5.23$ & $66.85 \pm 5.17$ & 85.71 & 85.71 \\
\hline
\end{tabular}

(1) Data are expressed as percentage of positive staining. (2) Asterisks $(*)$ indicate that the expression levels of CD68 did not exist statistically significant with respect to released patients with CML-AP group and CML-CP $(P>0.01)$. There was statistical significance among the other group and control group $(P<$ $0.01)$.

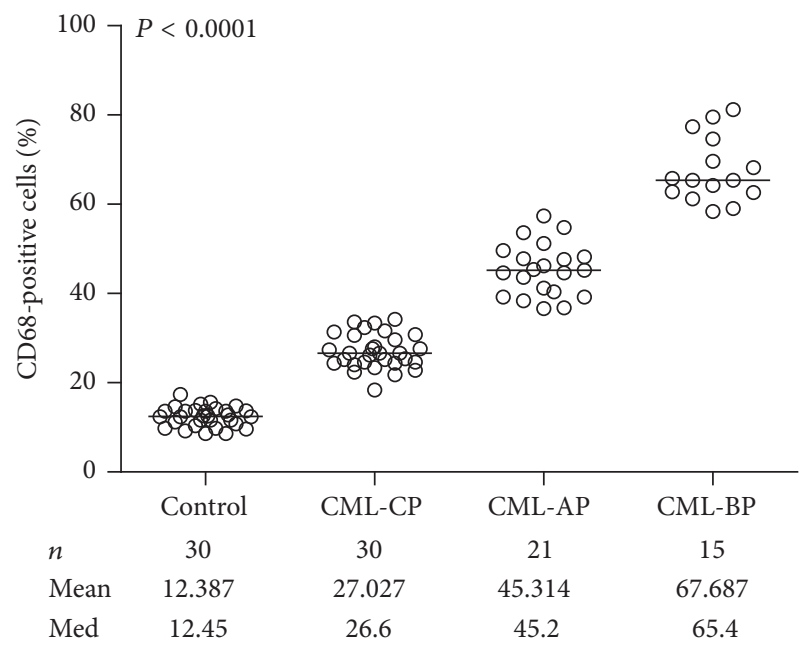

(a)

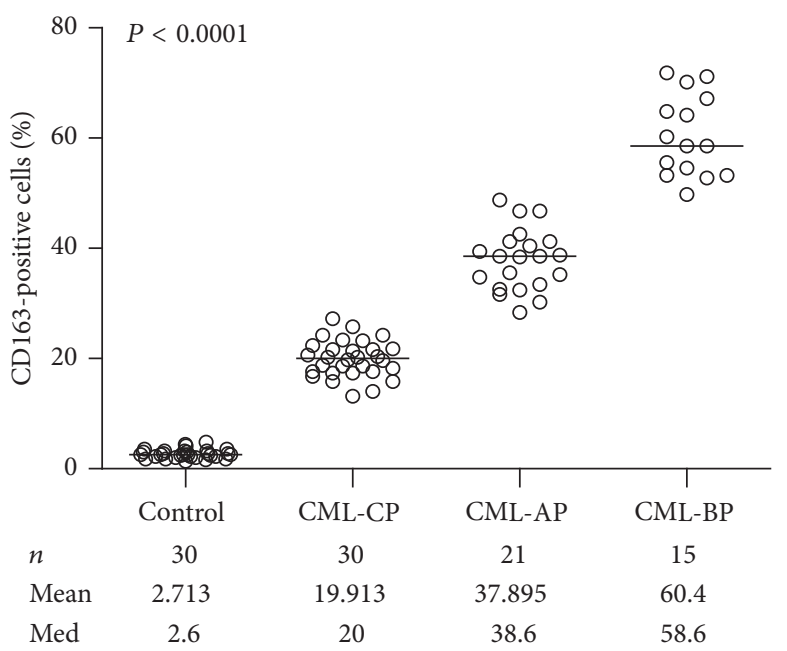

(b)

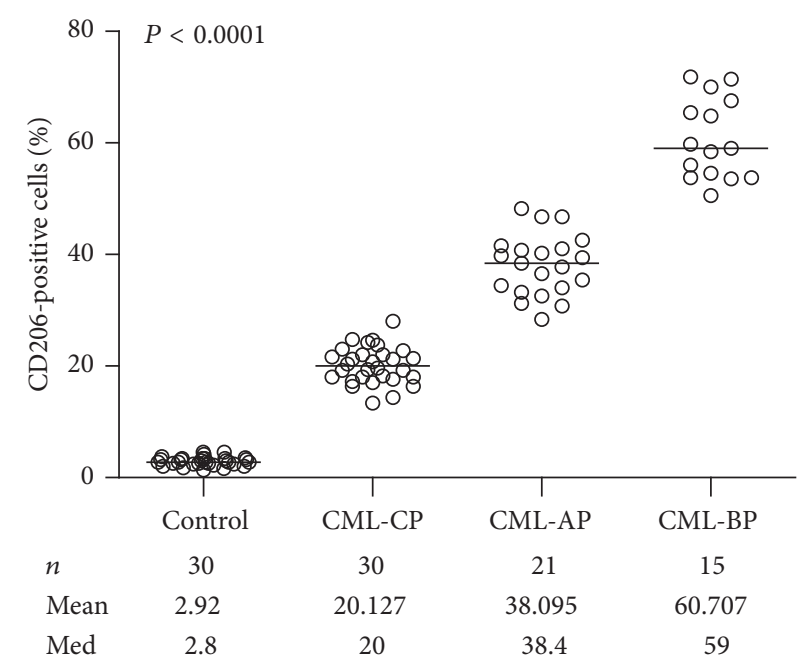

(c)

FIGURE 4: Analysis of infiltrating macrophages in BMB samples of CML patients. The percentage of macrophages positive for CD68 (a), CD163 (b), and CD206 (c) correlated with the development of CML. 


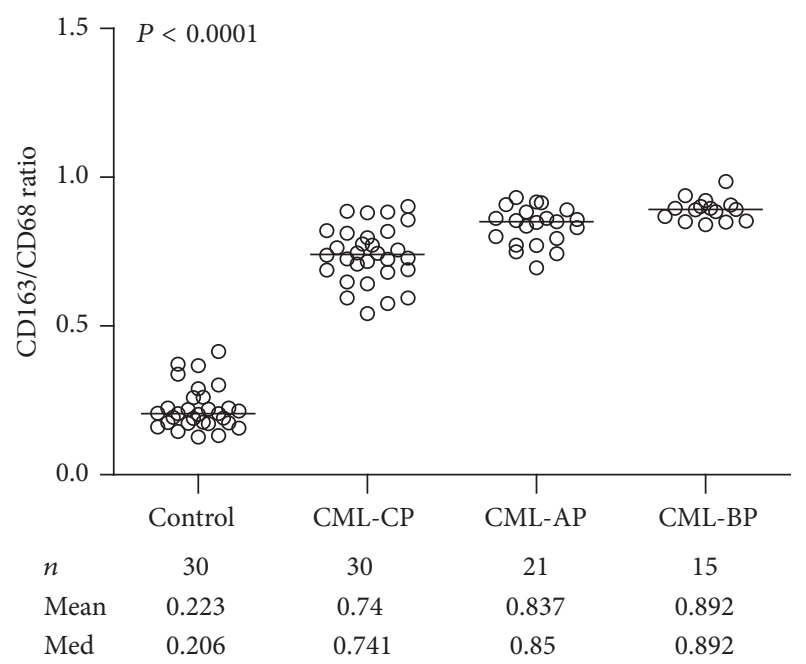

(a)

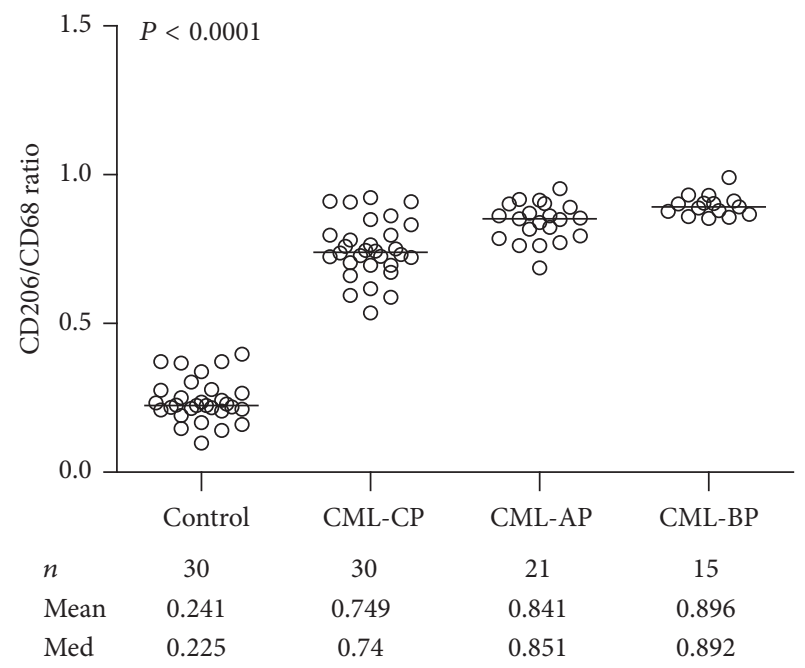

(b)

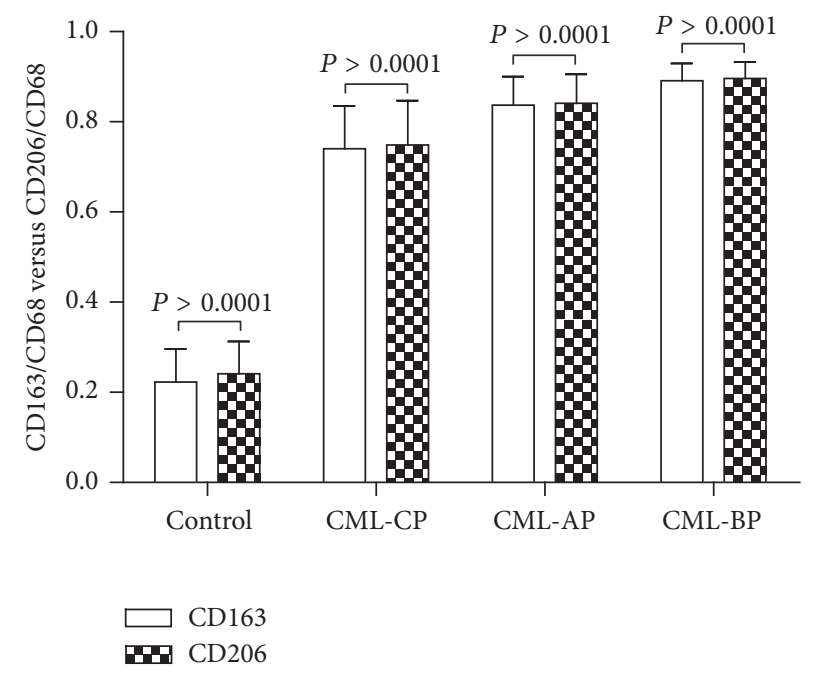

(c)

FIGURE 5: Analysis of infiltrating macrophages in BMB samples of CML patients. The proportions of CD163-positive (a) and CD206-positive (b) cells among CD68-positive cells associated with the different phase of CML. There was no difference between the positive expression ratio of CD163 and CD206 to CD68 (c) at the same phase.

proportion of CD163 and CD206-positive cells to CD68positive cells at the same stage (Figure 5(c)). It was decreased in the released patients with CML-CP (45.77\%), CML-AP (66.90\%), and CML-BP groups (78.27\%) (Table 2). In contrast to the control group, there existed statistically significant $(P<$ 0.01 ).

\section{Discussion}

Macrophages, a remarkably heterogeneous population, played an important role in tumor biology from early carcinogenesis to tumor progression including metastases [9-11]. However, the role of macrophages in CML remains to be elucidated. To examine whether expression of macrophages markers CD68, CD163, and CD206 associated with the CML development, immunohistochemical staining was performed. In this work we provide evidence of its counts and phenotype in $\mathrm{BMB}$ samples of CML patients. Further we show that a high frequency of $\mathrm{CD} 68^{+}, \mathrm{CD} 163^{+}$, and $\mathrm{CD} 206^{+}$ macrophages associated with the CML progression.

CD68 expressed on all macrophages has been widely used as a pan-macrophage marker; CD163 and CD206 are particular in the M2 phenotype [14, 19]. Our data show that the higher expression of CD68, CD163, and CD206 in $\mathrm{BMB}$ samples is a remarkable phenomenon during the transformation of $\mathrm{CP}$ to $\mathrm{AP}$ and $\mathrm{BP}$ in $\mathrm{CML}$ patients in contrast to those in control group. Moreover, in this study, the change of the macrophages phenotype was determined in CML development, which indicates that BM microenvironments imbalance, in CML patients, was towards M2like macrophages. This revealed that leukemia cells' survival and disease progression were associated with high counts of macrophages, particular in M2-like macrophages. Numerical increase in TAMs may be possibly related to enhancing 
phagocytic activity regarding the degradation of leukemia cells as well as to immune escape $[17,20]$. The previous reports have indicated that the $\mathrm{CD}^{+} 8^{+}$macrophages were increasing in BM of CML $[21,22]$, but the significance of CD163 and CD206 is expressed on BMB samples and it is associated with phases development of CML patients that has not been explored.

Markers of the M2 phenotype include CD163, CD184, CD204, CD206, CD209, receptors SR-A and M60, and so forth $[23,24]$. The proteins of both CD163 and CD206 are distributed in the membranes. However, there are slight differences between CD206-positive and CD163-positive M2 macrophages. M2 macrophages appear to be a heterogeneous population including $\mathrm{M} 2 \mathrm{a}, \mathrm{M} 2 \mathrm{~b}$, and $\mathrm{M} 2 \mathrm{c}$ in response to stimuli provided by local microenvironment. M2a subpopulations express high levels of CD206 upregulated by IL-4 or IL-13. M2b is induced by immune complexes and TLRs or IL$1 \mathrm{R}$. The roles of $\mathrm{M} 2 \mathrm{a}$ and $\mathrm{M} 2 \mathrm{~b}$ are immunoregulatory. $\mathrm{M} 2 \mathrm{c}$ is induced by IL-10 and glucocorticoids. M2c has the expression of CD163 and takes part in tissue remodeling [25]. We observed that CD163+ and CD206+ M2-like TAMs increased as the stages progressed in BMB samples of CML patients. But it still not completely understood their functional roles during phenotype changes and how M2-like macrophages cross talk to leukemia cells.

Leukemia cells are largely controlled by specific local microenvironments (i.e., "niches") and require a suitable microenvironment to maintain their growth [26]. However, the factors and mechanisms providing by bone marrow (BM) microenvironment are not fully understood [27, 28]. $\mathrm{BM}$ microenvironment comprised a variety of immune or nonimmune stromal cells like macrophages, adipose cells, mesenchymal stem cells and reticular cells, and so forth. Macrophages are the hot spots of cancer research [7-11]. TAMs are highly plastic dictated by their microenvironment conceptualized into two opposing but complementary activation states: either proinflammatory M1-like TAMs or anti-inflammatory M2-like TAMs [29]. M2-like TAM are alternatively activated and produce anti-inflammatory cytokines like IL-4, IL-10, IL-13, transforming growth factorb (TGF-b), and various chemokines to turn off damaging immune system activation and get involved in proliferation and survival of leukemia stem cell (LSCs), progression and metastasis, facilitating angiogenesis and lymphangiogenesis, matrix remodeling, and tumor cell-motility [30, 31]. Our results showed that $\mathrm{M} 2$-like macrophages were the major components of infiltrating TAMs that orchestrate various aspects of leukemia cells. In the beginning of CML, TAMs were the phenotype of the proinflammatory M1-like TAMs taking part in the antitumor. With the CML development, BM inflammation microenvironment induced the higher numbers of total TAMs, especially in M2-like TAMs. The balance of M1-like TAMs over M2-like TAMs was shifted towards the anti-inflammatory M2-like TAMs. Nonetheless upregulation of CD163 and CD206 is specific to M2-like TAM adaptation towards inflammation shown in BM of CML patients. The numbers of TAM, especially M2 positively phenotype, correlate with the differential stages of disease development of CML patients, which associated to the complex and multidirectional interaction between BM microenvironment and leukemia cells. We speculated that there exists a vicious connection between macrophages and cancer.

M2-like macrophages as their high expression get involved in the suppression of antitumor immune responses [7-11, 18-20]. Some articles described that M2-like macrophages can be converted to M1-like cells and reduced the immunosuppressive effects following IFN- $\gamma$ or other agent's treatment [17, 31-35]. It is suggested that new possible treatment strategies targeting M2-like macrophages in CML are round the corner.

TAM infiltration density in the release group was decreased in BMB samples of CML. In contrast to the control group, there existed statistical significance. It is suggested that the pathogenic factors involved in promoting leukemia cell exaggerated proliferation, differentiation blocked, and apoptosis resistance still existed, so the therapy strategy of CML needed to combine with modification bone marrow (BM) homeostasis $[5,36]$.

Macrophages are described as part of the mononuclear phagocyte system [37]. The developmental origin of macrophages has bone marrow hematopoiesis derived monocytes, yolk sac macrophages, and fetal monocytes [38-40]. Each tissue throughout the body has its own macrophages including resident macrophages and circulation macrophages [41]. In response to inflammation, infection, and tissue injury, macrophages are recruited into lesions $[42,43]$. We observed the expansion of macrophages in $\mathrm{BM}$ of CML patients, but whether monocyte recruitment or proliferation of resident or migration of other tissue's registration macrophages needs to be further identified.

\section{Conclusion}

The present study thus highlights the importance of positive expression of CD68, CD163, and CD206 increased in BM localization during chronic to blast phase transition of CML. The positive percentage ratios of CD163 and CD206 to CD68 are roaring upregulation, so targeting M2-like macrophages may help designing therapeutic strategies for CML. However, the reasons that resulted in accelerated macrophage expansion and increased M2-macrophages require in-depth studies.

\section{Competing Interests}

The authors would be happy to inform that there are no competing interests in producing the paper and there is no part in the paper that requires permission.

\section{Acknowledgments}

The authors would like to thank the entire staff and project members involved for their cooperation in this research project. This study received funding from the National Science Funding of China (NSFC no. 31460298) and Basic Research on Application of Joint Special Funding of Science 
and Technology Department of Yunnan Province, Kunming Medical University (nos. 2014FZ070, 2014FB092).

\section{References}

[1] S. Soverini, C. De Benedittis, M. Mancini, and G. Martinelli, "Best practices in chronic myeloid leukemia monitoring and management," The Oncologist, vol. 21, no. 5, pp. 626-633, 2016.

[2] S. Mukherjee and M. Kalaycio, "Accelerated phase CML: outcomes in newly diagnosed vs. Progression from chronic phase," Current Hematologic Malignancy Reports, vol. 11, no. 2, pp. 8693, 2016.

[3] K. Machová Polaková, T. Lopotová, H. Klamová et al., "Expression patterns of microRNAs associated with CML phases and their disease related targets," Molecular Cancer, vol. 10, article 41, 2011.

[4] L. Chiossone, R. Conte, G. M. Spaggiari et al., "Mesenchymal stromal cells induce peculiar alternatively activated macrophages capable of dampening both innate and adaptive immune responses," Stem Cells, vol. 34, no. 7, pp. 1909-1921, 2016.

[5] F. Peyrusson, D. Butler, P. M. Tulkens, and F. Van Bambeke, "Cellular pharmacokinetics and intracellular activity of the novel peptide deformylase inhibitor GSK1322322 against Staphylococcus aureus laboratory and clinical strains with various resistance phenotypes: studies with human THP-1 monocytes and J774 murine macrophages," Antimicrobial Agents and Chemotherapy, vol. 59, no. 9, pp. 5747-5760, 2015.

[6] M. Rath, I. Müller, P. Kropf, E. I. Closs, and M. Munder, "Metabolism via arginase or nitric oxide synthase: two competing arginine pathways in macrophages," Frontiers in Immunology, vol. 5, article 532, 2014.

[7] Y. Liu, K. Chen, C. Wang et al., "Cell surface receptor FPR2 promotes antitumor host defense by limiting M2 polarization of macrophages," Cancer Research, vol. 73, no. 2, pp. 550-560, 2013.

[8] M. Cieslewicz, J. Tang, J. L. Yu et al., "Targeted delivery of proapoptotic peptides to tumor-associated macrophages improves survival," Proceedings of the National Academy of Sciences of the United States of America, vol. 110, no. 40, pp. 15919-15924, 2013.

[9] X. Tang, C. Mo, Y. Wang, D. Wei, and H. Xiao, "Anti-tumour strategies aiming to target tumour-associated macrophages," Immunology, vol. 138, no. 2, pp. 93-104, 2013.

[10] Y. Komohara, M. Jinushi, and M. Takeya, "Clinical significance of macrophage heterogeneity in human malignant tumors," Cancer Science, vol. 105, no. 1, pp. 1-8, 2014.

[11] M. Heusinkveld and S. H. van der Burg, "Identification and manipulation of tumor associated macrophages in human cancers," Journal of Translational Medicine, vol. 9, no. 1, article 216, 2011.

[12] Y. Komohara, Y. Fujiwara, K. Ohnishi, D. Shiraishi, and M. Takeya, "Contribution of macrophage polarization to metabolic diseases," Journal of Atherosclerosis and Thrombosis, vol. 23, no. 1, pp. 10-17, 2016.

[13] F. O. Martinez, L. Helming, R. Milde et al., "Genetic programs expressed in resting and IL-4 alternatively activated mouse and human macrophages: similarities and differences," Blood, vol. 121, no. 9, pp. e57-e69, 2013.
[14] K. Kambara, W. Ohashi, K. Tomita et al., "In vivo depletion of CD206+ M2 macrophages exaggerates lung injury in endotoxemic mice," American Journal of Pathology, vol. 185, no. 1, pp. 162-171, 2015.

[15] J. Thiele, H. M. Kvasnicka, D. W. Beelen et al., "Macrophages and their subpopulations following allogeneic bone marrow transplantation for chronic myeloid leukaemia," Virchows Archiv, vol. 437, no. 2, pp. 160-166, 2000.

[16] S. K. Biswas and A. Mantovani, "Macrophage plasticity and interaction with lymphocyte subsets: cancer as a paradigm," Nature Immunology, vol. 11, no. 10, pp. 889-896, 2010.

[17] J. Thiele, C. Braeckel, S. Wagner et al., "Macrophages in normal human bone marrow and in chronic myeloproliferative disorders: an immunohistochemical and morphometric study by a new monoclonal antibody (PG-M1) on trephine biopsies," Virchows Archiv A-Pathological Anatomy and Histopathology, vol. 421, no. 1, pp. 33-39, 1992.

[18] Y. Komohara, K. Ohnishi, J. Kuratsu, and M. Takeya, "Possible involvement of the M2 anti-inflammatory macrophage phenotype in growth of human gliomas," Journal of Pathology, vol. 216, no. 1, pp. 15-24, 2008.

[19] Y. Komohara, D. Niino, K. Ohnishi, K. Ohshima, and M. Takeya, "Role of tumor-associated macrophages in hematological malignancies," Pathology International, vol. 65, no. 4, pp. 170-176, 2015.

[20] D. Duluc, Y. Delneste, F. Tan et al., “Tumor-associated leukemia inhibitory factor and IL-6 skew monocyte differentiation into tumor-associated macrophage-like cells," Blood, vol. 110, no. 13, pp. 4319-4330, 2007.

[21] A. Georgii, K. F. Vykoupil, T. H. Buhr et al., "Chronic myeloproliferative disorders in bone marrow biopsies," Pathology Research and Practice, vol. 186, no. 1, pp. 3-27, 1990.

[22] G. Büsche, H. Majewski, J. Schlué et al., "Frequency of pseudoGaucher cells in diagnostic bone marrow biopsies from patients with Ph-positive chronic myeloid leukaemia," Virchows Archiv, vol. 430, no. 2, pp. 139-148, 1997.

[23] Y. Saito, Y. Komohara, D. Niino et al., "Role of CD204positive tumor-associated macrophages in adult T-cell leukemia/lymphoma," Journal of Clinical and Experimental Hematopathology, vol. 54, no. 1, pp. 59-65, 2014.

[24] P. J. Murray and T. A. Wynn, "Protective and pathogenic functions of macrophage subsets," Nature Reviews Immunology, vol. 11, no. 11, pp. 723-737, 2011.

[25] F. Ginhoux, J. L. Schultze, P. J. Murray, J. Ochando, and S. K. Biswas, "New insights into the multidimensional concept of macrophage ontogeny, activation and function," Nature Immunology, vol. 17, no. 1, pp. 34-40, 2016.

[26] M. M. Mohamed, E. A. El-Ghonaimy, M. A. Nouh, R. J. Schneider, B. F. Sloane, and M. El-Shinawi, "Cytokines secreted by macrophages isolated from tumor microenvironment of inflammatory breast cancer patients possess chemotactic properties," International Journal of Biochemistry and Cell Biology, vol. 46, no. 1, pp. 138-147, 2014.

[27] K. Suzuki, R. Sun, M. Origuch et al., "Mesenchymal stromal cells promote tumor growth through the enhancement of neovascularization," Molecular Medicine, vol. 17, no. 7-8, pp. 579-587, 2011.

[28] D. S. Krause, K. Fulzele, A. Catic et al., "Differential regulation of myeloid leukemias by the bone marrow microenvironment," Nature Medicine, vol. 19, no. 11, pp. 1513-1517, 2013.

[29] T. Salim, C. L. Sershen, and E. E. May, "Investigating the role of TNF- $\alpha$ and IFN- $\gamma$ activation on the dynamics of iNOS gene 
expression in LPS stimulated macrophages," PLoS ONE, vol. 11, no. 6, Article ID e0153289, 2016.

[30] O. M. Pello, M. De Pizzol, M. Mirolo et al., "Role of c-MYC in alternative activation of human macrophages and tumorassociated macrophage biology," Blood, vol. 119, no. 2, pp. 411421, 2012.

[31] I. N. Buhtoiarov, P. M. Sondel, J. M. Wigginton et al., "Antitumour synergy of cytotoxic chemotherapy and anti-CD40 plus CpG-ODN immunotherapy through repolarization of tumourassociated macrophages," Immunology, vol. 132, no. 2, pp. 226239, 2011.

[32] Y. Fujiwara, M. Takeya, and Y. Komohara, "A novel strategy for inducing the antitumor effects of triterpenoid compounds: Blocking the protumoral functions of tumorassociated macrophages via stat3 inhibition," BioMed Research International, vol. 2014, Article ID 348539, 11 pages, 2014.

[33] C. Ngambenjawong, M. Cieslewicz, J. G. Schellinger, and S. H. Pun, "Synthesis and evaluation of multivalent M2pep peptides for targeting alternatively activated M2 macrophages," Journal of Controlled Release, vol. 224, pp. 103-111, 2016.

[34] D. Duluc, M. Corvaisier, S. Blanchard et al., "Interferon$\gamma$ reverses the immunosuppressive and protumoral properties and prevents the generation of human tumor-associated macrophages," International Journal of Cancer, vol. 125, no. 2, pp. 367-373, 2009.

[35] C. Baer, M. L. Squadrito, D. Laoui et al., "Suppression of microRNA activity amplifies IFN- $\gamma$-induced macrophage activation and promotes anti-tumour immunity," Nature Cell Biology, vol. 18, no. 7, pp. 790-802, 2016.

[36] K. Hatogai, S. Kitano, S. Fujii et al., "Comprehensive immunohistochemical analysis of tumor microenvironment immune status in esophageal squamous cell carcinoma," Oncotarget, 2016.

[37] S. Lemaire, M.-P. Mingeot-Leclercq, P. M. Tulkens, and F. Van Bambeke, "Study of macrophage functions in murine J774 cells and human activated THP-1 cells exposed to oritavancin, a lipoglycopeptide with high cellular accumulation," Antimicrobial Agents and Chemotherapy, vol. 58, no. 4, pp. 2059-2066, 2014.

[38] F. Ginhoux and M. Guilliams, "Tissue-resident macrophage ontogeny and homeostasis," Immunity, vol. 44, no. 3, pp. 439449, 2016.

[39] E. Gomez Perdiguero, K. Klapproth, C. Schulz et al., "Tissueresident macrophages originate from yolk-sac-derived erythromyeloid progenitors," Nature, vol. 518, no. 7540, pp. 547-551, 2015.

[40] S. Epelman, K. J. Lavine, and G. J. Randolph, "Origin and functions of tissue macrophages," Immunity, vol. 41, no. 1, pp. 21-35, 2014.

[41] A. Dey, J. Allen, and P. A. Hankey-Giblin, "Ontogeny and polarization of macrophages in inflammation: blood monocytes versus tissue macrophages," Frontiers in Immunology, vol. 5, article 683, 2014.

[42] R. Gentek, K. Molawi, and M. H. Sieweke, “Tissue macrophage identity and self-renewal," Immunological Reviews, vol. 262, no. 1, pp. 56-73, 2014.

[43] R. A. Franklin and M. O. Li, "Ontogeny of tumor-associated macrophages and its implication in cancer regulation," Trends Cancer, vol. 2, no. 1, pp. 20-34, 2016. 


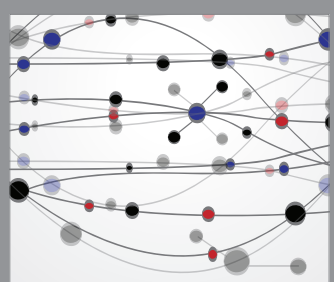

The Scientific World Journal
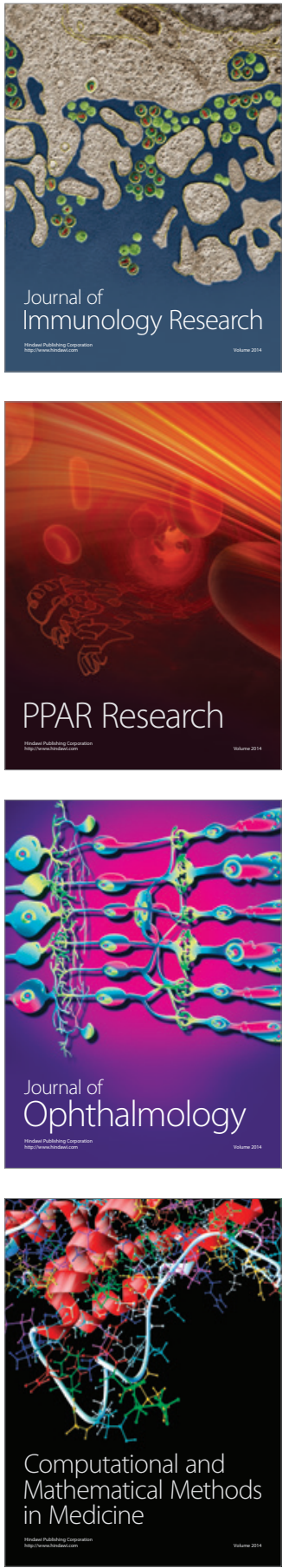

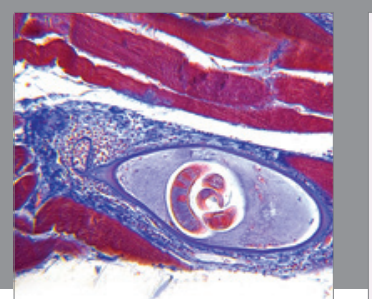

Gastroenterology Research and Practice

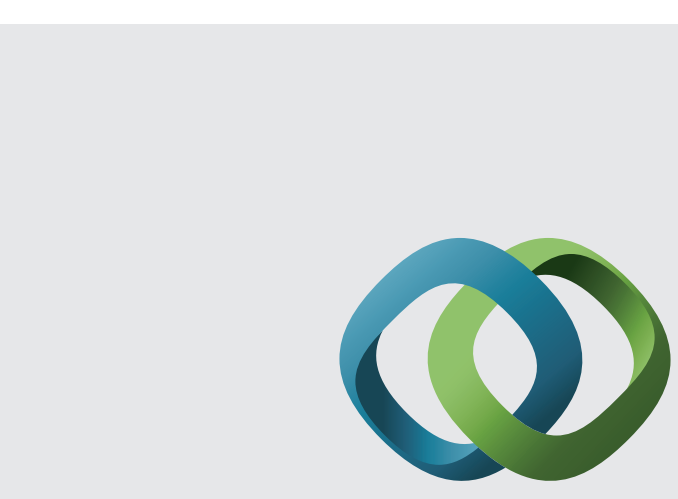

\section{Hindawi}

Submit your manuscripts at

http://www.hindawi.com
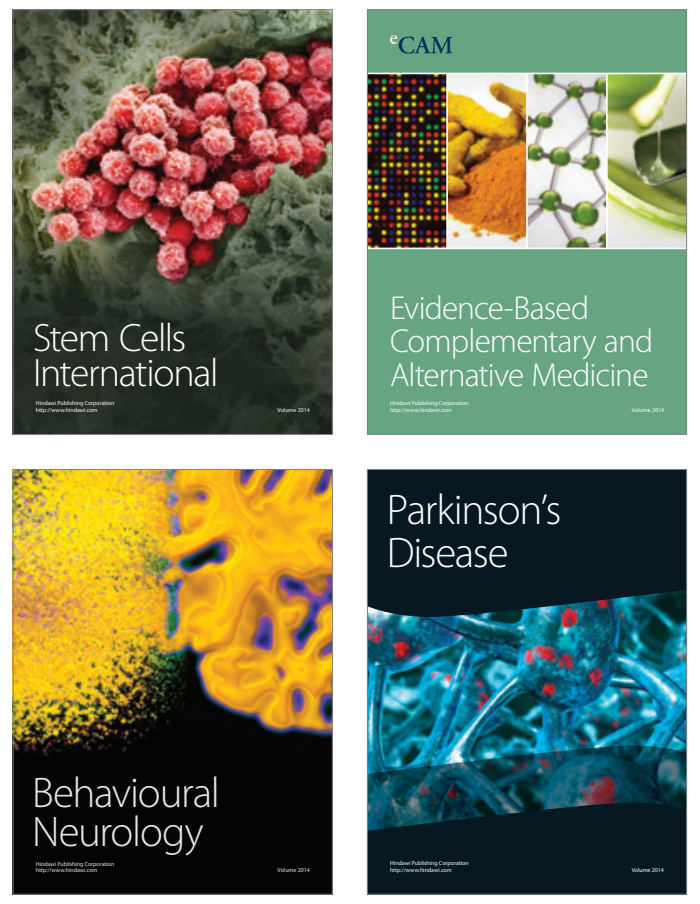
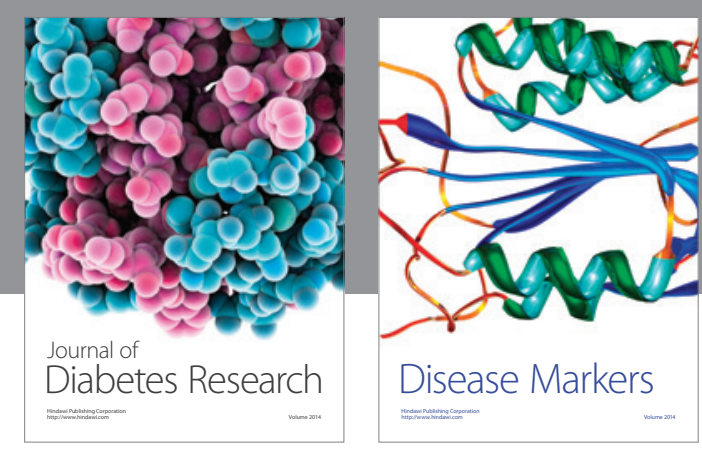

Disease Markers
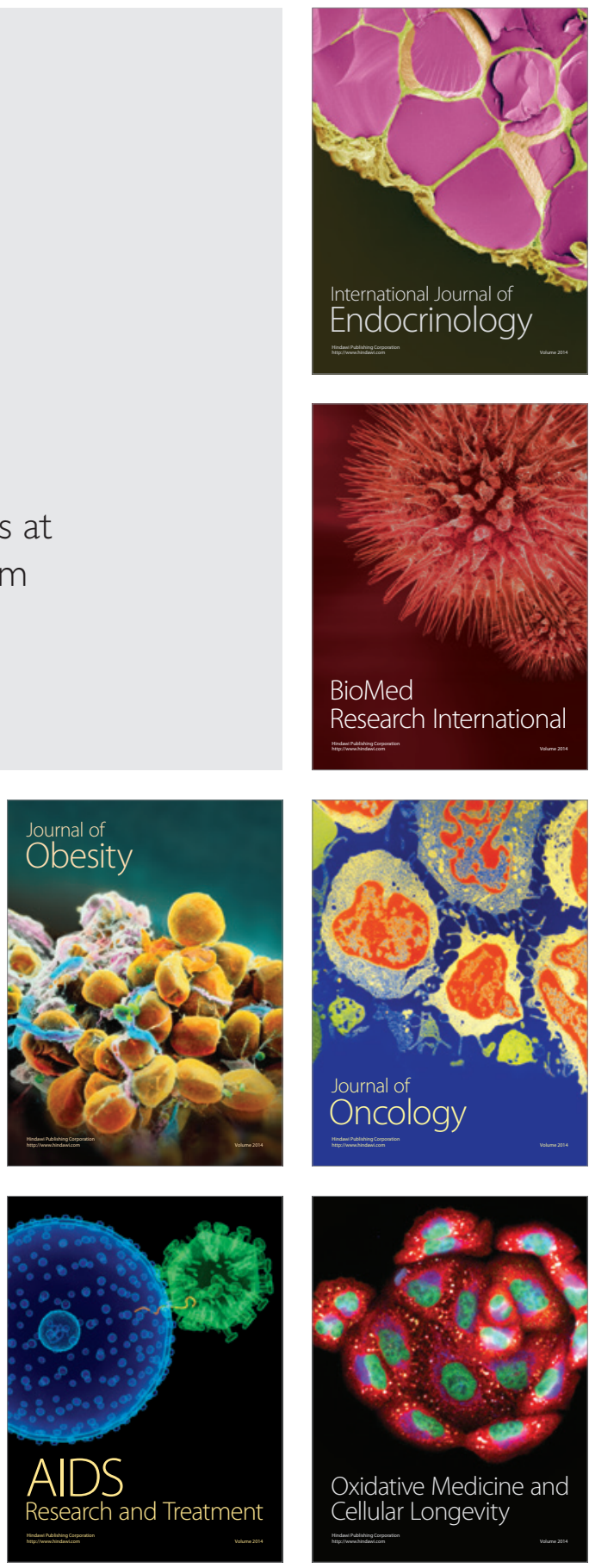